\title{
Distributed Maintenance of a Spanning Tree using Labeled Tree Encoding
}

\author{
Vijay K. Garg * \\ Department of Electrical and Computer Engineering \\ The University of Texas at Austin \\ Austin, TX 78712-1084, USA \\ garg@ece.utexas.edu
}

\author{
Anurag Agarwal \\ Department of Computer Sciences \\ The University of Texas at Austin \\ Austin, TX 78712-0233, USA \\ anurag@cs.utexas.edu
}

\begin{abstract}
Maintaining spanning trees in a distributed fashion is central to many networking applications and selfstabilizing algorithms provide an elegant way of doing it in fault-prone environments. In this paper, we propose a self-stabilizing algorithm for maintaining a spanning tree in a distributed fashion for a completely connected topology. Our algorithm requires a node to process $O(1)$ messages on average in one asynchronous round as compared to previous algorithms which need to process messages from every neighbor, resulting in $O(n)$ work in a completely connected topology. Our algorithm also stabilizes faster than the previous approaches. Our approach demonstrates a new methodology which uses the idea of core and non-core states for developing self-stabilizing algorithms. The algorithm is also useful in security related applications due to its unique design.
\end{abstract}

\section{Introduction}

Fault tolerance is a major concern in distributed systems. The self-stabilization paradigm, introduced by Dijkstra [Dij74], is an elegant and a powerful mechanism to handle one particular class of faults. These faults are the transient faults which can corrupt the state of the system and will be called data faults. Self-stabilizing systems ensure that a system starting from any state would converge to a legal state provided the faults cease to occur.

* supported in part by the NSF Grants ECS-9907213, CCR9988225, Texas Education Board Grant ARP-320, an Engineering Foundation Fellowship, and an IBM grant.
Spanning trees have many uses in computer networks. Once a spanning tree is established in a network, it may be used in broadcast of a message, convergecast, $\beta$ synchronizer, and many other algorithms. As a result, it is desirable to have an efficient self-stabilizing algorithm for spanning trees. Self-stabilizing algorithms for spanning tree construction have been extensively studied. The first algorithm in this area was given in [DIM89, DIM90] which deals with building BFS tree for a graph. Other algorithms were also proposed for self-stabilizing BFS trees which dealt with different system models and assumptions [AKY91],[AG94], [HC92]. Algorithms have also been proposed for other types of trees - like DFS tree [CD94] and minimum spanning tree [AS97]. There have been other papers which try to optimize on the memory used and stabilization time [Joh97], [AK93].

In this paper, we demonstrate a new technique for constructing self-stabilizing algorithms by using it for maintaining spanning trees. It is well known that one can design self-stabilizing algorithms with detection and reset strategy [AG94]. In this strategy, the nodes periodically test if the system is in legal state and on detection of a fault, carry out the reset strategy. Many self-stabilizing algorithms have local detection, i.e., the detection by each node corresponds to evaluation of a boolean predicate only on its and its neighbors' variables. The reset procedure may be complicated depending upon the application.

Our method is an extension of the above strategy. We view the set of global states as cross-product of core states and non-core states. The core states satisfy the property: There exists a legal state for every core state. The non-core component of a global state is maintained only for performance reason. Given the core component, 
one could always recreate the non-core component. In our algorithm for maintaining a spanning tree, we will use Neville's code [Nev53] of the tree as the core component and parent structure as the non-core component. Given any Neville's code, there exists a unique labeled spanning tree in a completely connected graph. Now assume that our program suffers from a data fault. The data fault could be in the core component or the non-core component. The crucial property that we use is that every bit pattern in the core component results in a valid code. Therefore, in either case, we will assume that it is the non-core component that has changed. If there is an efficient method of detecting that the non-core component does not correspond to the core component, we simply reset the non-core component to a value corresponding to the core component. The challenge lies in efficient detection and reset of the state when information is distributed across the network. Similarly, for maintaining a permutation, its inversion vector can be the core component and then some auxiliary data structures can be maintained to make the fault detection and correction efficient. Details of this example are given in the full version of this paper [GA04].

We assume our system to be a completely connected graph with $n$ nodes having ids from 1 to $n$. Our algorithm is designed for asynchronous message-passing systems, and unlike many other self-stabilizing algorithms, it does not require a central daemon [Dij74] for scheduling decisions. Although some of our assumptions are stronger than the previous work, our algorithm has some significant advantages. Traditionally, the notion of time used in asynchronous algorithms is the number of asynchronous rounds [DIM90] it executes. Previous algorithms require examining every neighbor's variables during correction, needing $O(n)$ asynchronous rounds in one asynchronous cycle [DIM90] for a completely connected topology. Our algorithm does not fit in well with model of asynchronous rounds due to presence of asynchronous receives. We use a model similar to synchronous model for evaluating the time complexity of our algorithm. In this model, the stabilization time of the previous algorithms remains the same as that in asynchronous rounds model. In the new model, our algorithm requires every process to handle only $O(1)$ messages on average in one cycle. The stabilization time of our algorithm is $O(d)$, where $d$ is an upper bound on the number of times a node appears in the Neville's code. It turns out that $d$ is $O((\log n) / \log \log n)$ with very high probability for a randomly chosen code. This gives a very small stabilization time. Moreover, as a result of using the idea of core and non-core states, we also provide the individual nodes with the ability to systematically change the structure of the tree. This renders the algorithm useful in settings not traditionally associated with self-stabilizing systems. As an example, we consider an application where the participating nodes would like to periodically change the structure of the tree in a distributed fashion for security purposes. This can be done using our algorithm by changing code value for some node which would result in a different tree upon stabilization. Another useful feature of our algorithm is that it allows the root of the tree to change dynamically. This is different from most of the previous approaches where the root node executes a different algorithm from the rest of the nodes, resulting in a fixed root. We also discuss an application which requires this feature. In summary, the main advantages of our algorithm are:

- Fast stabilization.

- Allows systematic change in tree structure.

- Root node can change dynamically.

\section{Neville's Third Encoding}

We assume that processes are labeled as $P_{1}, \ldots, P_{n}$ and they form a completely connected graph. To maintain a spanning tree (in a non-stabilizing manner), it is sufficient for each process to maintain the parent variable but this method is not self-stabilizing as a fault in the parent pointer of some process may result in an invalid structure.

For simplicity we assume that all spanning trees rooted at $P_{n}$ constitute the set of legal structures. Later we explain how this assumption can be relaxed to allow any node to become the root. We represent a tree through an encoding for labeled trees called the Neville's third encoding [Nev53] (The reader can find a discussion on other labeled tree encodings and their properties in [DM01]). In this paper the term "Neville's code" refers to Neville's third code. Each labeled spanning tree has a one-to-one correspondence with a Neville's code. This code is a sequence of $n-2$ numbers from the set $\{1 \ldots n\}$. Let Neville's code of the tree be denoted by code $[i]$ for $i \in\{1 \ldots n-2\}$. For completeness sake, derivation of Neville's code from a labeled spanning tree is discussed. 
Given a labeled spanning tree with $n$ nodes, the Neville's code can be obtained by deleting $n-1$ edges in the tree as shown in Figure 1.

$$
\begin{aligned}
& x[1]=\text { least node with degree } 1 \\
& \text { for }(i=1 ; i<n ; i++) \\
& y[i]=\text { neighbor of } x[i] \\
& \text { delete edge between } x[i] \text { and } y[i] \\
& \text { if }(\text { degree }[y[i]]==1) \\
& \quad x[i+1]=y[i] \\
& \text { else } \\
& x[i+1]=\text { least node with degree } 1
\end{aligned}
$$

Figure 1: Algorithm to compute Neville's code of a labeled tree

The algorithm starts by deleting the least leaf node (leaf with least label). In iteration $i$, a node $x[i]$ is deleted and its neighbor $y[i]$ is recorded. The edge between $x[i]$ and $y[i]$ is deleted as well. Then, variable $x[i+1]$ is set to $y[i]$ if the degree of $y[i]$ is 1 , otherwise it is set to the least leaf node. The sequence $\{y[i] \mid 1 \leq i \leq n-2\}$, thus obtained, is called Neville's code. Note that even though there are $n-1$ iterations, we only consider $n-2$ entries as the value of $y[n-1]$ is always $n$. For all the algorithms in this paper we consider the $n-1$ length code which explicitly includes $n$ at the end. As an example, consider the labeled tree given in Figure 2. To compute the Neville's code for the tree, we start by deleting the least leaf node, 1. Since the parent of 1 is 5 , at this point the code is (5). Now 5 is still not a leaf, so we again choose the least leaf node in the remaining tree, 3 . We proceed by deleting 3 and adding its parent 2 to the code. Continuing in a similar fashion, after $n-1=6$ iterations of the algorithm, the code $(5,2,7,5,5,7)$ is obtained.

Given Neville's code, the labeled spanning tree can also be computed easily. We first calculate the degree of each node $v$ in the labeled spanning tree as follows: degree $(v)=1+$ number of times $v$ appears in the code. Note that for the root node $n$, this gives a value which is one higher than the actual degree of the root but this is required for the correctness of the algorithm. Once degree of each node is known, the procedure given in Figure 3 can be used to compute the code.

We require $P_{i}$ to maintain code $[i]$ as the core data structure and parent $[i]$ as the non-core data structure. If efficiency were not an issue, this would be sufficient for a self-stabilizing algorithm. Periodically, all nodes would

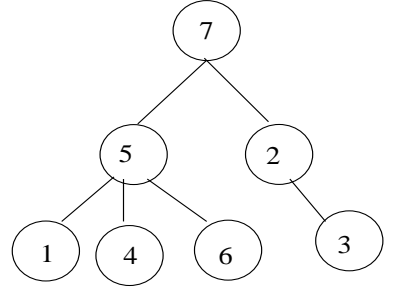

Figure 2: A spanning tree with Neville's code $(5,2,7,5,5,7)$

$$
\begin{aligned}
& j=\text { least node with degree } 1 \\
& \text { for }(i=1 ; i<n ; i++) \\
& \text { parent }[j]=\operatorname{code}[i] \\
& \text { degree }[j]-- \\
& \text { degree }[\operatorname{code}[i]]-- \\
& \text { if }(\operatorname{degree}[\operatorname{code}[i]]==1) \text { then } \\
& j=\operatorname{code}[i] \\
& \text { else } \\
& j=\text { least degree node with degree } 1
\end{aligned}
$$

Figure 3: Algorithm to compute labeled tree from Neville's code

send their code to $P_{n}$. $P_{n}$ would calculate parent $[i]$ for each node $P_{i}$ and send it back. $P_{i}$ would reset parent $[i]$ to the value received from $P_{n}$. Even if parent $[i]$ was corrupted, it would be reset to agree with the spanning tree given by Neville's code. If the variable code $[i]$ gets changed, it would still result in a valid spanning tree. The parent pointers would then be reset to agree with the new code.

This method would be wasteful when there are no errors. The code and the parent values would be exchanged without serving any purpose. In a large network, it is desirable to have local detection of error and only on an error, the correction algorithm should be invoked.

\section{Non-Core Data Structures for Spanning Trees}

Our strategy would be to introduce new data structures in the system so that by imposing a set of constraints on these data structures, we can efficiently detect and correct data faults. For this purpose, the following data struc- 
tures are used:

- parent: The variable parent $[i]$ gives the parent of node $P_{i}$ in the spanning tree.

- $f$ : The variable $f[i]$ gives us the iteration in which the node $P_{i}$ is deleted in the algorithm for obtaining Neville's code of a tree. Therefore, code $[f[i]]$ gives us the parent $[i]$. Since $P_{n}$ is not deleted in first $n-1$ iterations, we assume that $f[n]=n$.

- $z$ : The variable $z[i]$ gives the largest value of $j$ such that $\operatorname{code}[j]=i$. If there is no such $j$, then $z[i]=0$.

Based on the properties of Neville's code, it can be verified that the variables - code, parent, $f$ and $z-$ satisfy the following constraints:

(R1) $\forall i: \operatorname{code}[f[i]]=$ parent $[i]$

Follows from the property of the $f$ relating it to the parent.

(R2) $(\forall i: 1 \leq i \leq n-2 \Rightarrow 1 \leq \operatorname{code}[i] \leq n)$

$\wedge(\operatorname{code}[n-1]=n) \wedge(\operatorname{code}[n]=0)$

This constraint is the definition of code extended to all the nodes.

(R3) (i) $\forall i: 1 \leq i<n \Rightarrow 1 \leq f[i] \leq n-1$

This constraint puts restriction on the range of values that a node other than the root is allowed to take.

(ii) $f$ is a permutation on $[1 \ldots n]$

The definition of $f$ along with the topology of the algorithm in Figure 1 imply that the $f$ values are distinct and range from $1 \ldots n$.

(R4) $\forall j: z[j]=\max \{i \mid \operatorname{code}[i]=j\} \cup\{0\}$

This is the definition of $z$.

(R5) $\forall i: z[i] \neq 0 \Rightarrow(f[i]=z[i]+1)$

If a node $i$, which at the starting of the algorithm was not a leaf node, becomes a leaf node during the iteration $j$ of the algorithm, then it is deleted in the iteration $j+1$. This constraint enforces this condition.

These constraints are strong enough to characterize a spanning tree, i.e., given a set of data structures code, parent, $f$ and $z$ which satisfy these constraints, the parent structure results in a valid spanning tree regardless of the definitions of these data structures. From now on, when we consider the data structures code, parent, $f$ and $z$, we would just think of them as obeying a certain set of constraints and not necessarily corresponding to the original definitions that were given for them.

We would be dealing with two sets of constraints $-\mathcal{R}=\{R 1, R 2, R 3(i), R 4, R 5\}$ and $\mathcal{C}=$ $\{R 1, R 2, R 3, R 4, R 5\}$. It is evident that any algorithm which satisfies the constraint set $\mathcal{C}$ would also satisfy the constraint set $\mathcal{R}$. The trees resulting from obeying these constraint sets possess different guarantees. The two theorems that follow provide a characterization of those guarantees.

Theorem 1 If code, parent, $f$ and $z$ satisfy constraint set $\mathcal{R}$ then parent forms a valid spanning tree rooted at $P_{n}$.

Proof: Let the directed graph formed by the parent relation satisfying constraints $\mathcal{R}$ be $T_{\text {parent }}$. The edges of $T_{\text {parent }}$ are directed from the child to the parent.

We first show that $T_{\text {parent }}$ is acyclic. Let $i=$ parent $[j]$ in $T_{\text {parent }}$ for some nodes $i$ and $j$. Then,
code $[f[j]]=i$
$\Rightarrow(z[i] \neq 0) \wedge(f[j] \leq z[i])$
(Using (R1))
$\Rightarrow f[j]<f[i$
(Using (R4))
(Using (R5) for $i$ )

Applying this argument repeatedly shows that ancestor of a node has higher $f$ value than the $f$ value for the node itself. This implies that no node is ancestor of itself and hence $T_{\text {parent }}$ is acyclic.

We now show that every node except $P_{n}$ has outdegree 1 and $P_{n}$ has outdegree 0 . Consider a node $i \neq n$. Then,
$f[i] \neq n$
(Using (R3)(i))
$\Rightarrow 1 \leq \operatorname{code}[f[i]] \leq n$
(Using (R2))
$\Rightarrow 1 \leq$ parent $[i] \leq n$
(Using (R1))

This implies that in $T_{\text {parent }}$, every node except $P_{n}$ has outdegree 1. For $P_{n}$, consider the following:

$$
\begin{array}{ll}
\text { code }[n-1]=n \wedge \text { code }[n]=0 & \text { (Using (R2)) } \\
\Rightarrow z[n]=n-1 & \text { (Using (R4)) } \\
\Rightarrow f[n]=z[n]+1 & \text { (Using (R5)) } \\
\Rightarrow f[n]=n & \\
\Rightarrow \text { code }[f[n]]=0 & \text { (Using (R2)) } \\
\Rightarrow \text { parent }[n]=0 & \text { (Using (R1)) }
\end{array}
$$

Therefore, $P_{n}$ does not have a parent. Since all other nodes have a parent within the range $1 \ldots n$ and there are no cycles in $T_{\text {parent }}, T_{\text {parent }}$ forms a spanning tree rooted at $P_{n}$.

The above theorem just ensures that the parent forms a spanning tree. It does not enforce any relationship be- 
tween the structure of the tree formed by parent and tree corresponding to code. The next theorem establishes this relationship.

Theorem 2 If code, parent, $f$ and $z$ satisfy constraint set $\mathcal{C}$, then parent forms a rooted spanning tree isomorphic to the tree generated by code.

Proof: Since code, parent, $f$ and $z$ satisfy the constraint set $\mathcal{C}$, they also satisfy the constraint set $\mathcal{R}$. Hence, by Theorem 1, parent forms a spanning tree rooted at $P_{n}$.

Here we would be dealing with two trees:

(1) $T_{\text {parent }}$ : The tree formed by parent which satisfies $C$ and

(2) $T_{\text {code }}$ : The tree generated using code by the algorithm given in Figure 3.

We define data structures parent ${ }^{\prime}, f^{\prime}$ and $z^{\prime}$ for $T_{\text {code }}$. Variable parent ${ }^{\prime}[i]$ represents the parent of node $i$ in $T_{\text {code }}, f^{\prime}[i]$ gives the iteration in which the node $i$ is assigned its parent during the execution of algorithm for building $T_{\text {code }}$ from code and $z^{\prime}[i]$ gives the last occurrence of $i$ in code. Since the constraint (R4) is the same as definition for $z^{\prime}, z^{\prime}$ is same $z$. Both $f$ and $f^{\prime}$ are permutations on $1 \ldots n$. This implies that $\forall i\left(\exists j: f[i]=f^{\prime}[j]\right)$ and moreover, this $j$ is unique. This allows us to define an isomorphism function, $M:[n] \rightarrow[n]$ as:

$$
M(i)=j \text { such that } f[i]=f^{\prime}[j]
$$

Now, $T_{\text {parent }}$ and $T_{\text {code }}$ are isomorphic iff

$$
\forall i, j:(i=\text { parent }[j]) \Rightarrow M(i)=\operatorname{parent}^{\prime}[M(j)]
$$

We prove the above condition by showing that $\forall i, j:(i=$ parent $[j]) \Rightarrow M(i)=i$ followed by proving that $\forall i, j:(i=$ parent $[j]) \Rightarrow i=$ parent $^{\prime}[M(j)]$. The data structures parent ${ }^{\prime}, f^{\prime}$ and $z$ obey the constraint set $C$. Consider a node $i=\operatorname{parent}[j]$ for some nodes $i$ and $j$. Then,

$$
\begin{array}{ll}
z[i] \neq 0 & (\text { Using (R1) and (R4)) } \\
\Rightarrow f^{\prime}[i]=z[i]+1 & \left(\text { Using (R5) for } f^{\prime} \text { and } z[i]=z^{\prime}[i]\right) \\
\Rightarrow f[i]=f^{\prime}[i] & (\text { Using (R5) for } f) \\
\Rightarrow M(i)=i & \text { (Definition of } M)-(1)
\end{array}
$$

Node $i$ also satisfies the following property:

$$
\begin{array}{ll}
i=\operatorname{code}[f[j]] & \text { (Using (R1) for } f \text { ) } \\
\Rightarrow i=\text { code }\left[f^{\prime}[M(j)]\right] & \text { (Definition of } M \text { ) } \\
\Rightarrow i=\operatorname{parent}^{\prime}[M(j)] & \left(\text { Using }(\mathrm{R} 1) \text { for } f^{\prime}\right)-(2)
\end{array}
$$

Conditions (1) and (2) together prove the required isomorphism condition and hence the two trees $T_{\text {parent }}$ and $T_{\text {code }}$ are isomorphic.

\begin{tabular}{|c|c|c|c|c|c|c|c|}
\hline$i$ & 1 & 2 & 3 & 4 & 5 & 6 & 7 \\
\hline parent & 2 & 7 & 5 & 5 & 7 & 5 & 0 \\
\hline code & 5 & 2 & 7 & 5 & 5 & 7 & 0 \\
\hline$f$ & 2 & 3 & 1 & 4 & 6 & 5 & 7 \\
\hline$z$ & 0 & 2 & 0 & 0 & 5 & 0 & 6 \\
\hline
\end{tabular}

Table 1: Example of structures parent, code, $f$ and $z$ satisfying the constraints (R1)-(R5)

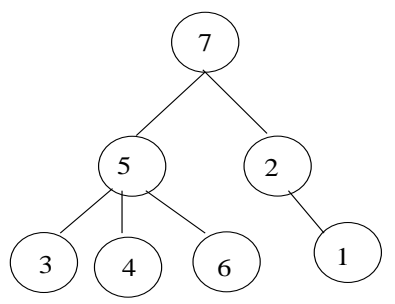

Figure 4: Tree corresponding to parent given in Table 1

The above theorem suggests that there is a possibility that the tree formed by parent is not the same as the tree generated by the code. For example, consider the value of the variables given in Table 1 . It can be easily verified that these values satisfy the constraint set $C$. The tree corresponding to the code is the one we considered earlier in Figure 2. The tree generated by parent is shown in Figure 4. The two trees are not the same but they are isomorphic.

\section{Maintaining Constraints}

Each node $i$ maintains parent $[i], \operatorname{code}[i], f[i]$ and $z[i]$ and cooperate to ensure that the required constraints are satisfied, resulting in a valid rooted spanning tree. We present a strategy for efficient detection and correction of the faults for each of the constraints.

\subsection{Constraints (R1) and (R2)}

The constraint (R1) is trivial to check locally. Each node $i$ inquires node $j=f[i]$ for $\operatorname{cod} e[j]$. If this value does not match parent $[i]$, then the constraint $(R 1)$ is violated. On violation, (R1) can be ensured by setting parent $[i]$ to code $[j]$. The constraint (R2) is also trivial to check and correct locally. 


\subsection{Constraint (R3)}

Constraint (R3)(i) is a local constraint which can be checked easily. Violation of this constraint can be fixed by simply setting $f$ to a random number between 1 and $n-1$. Constraint (R3)(ii) requires $f$ to be a permutation on $1 \ldots n$. This can in turn be modeled in terms of the following constraints:

(C1) $\forall i: 1 \leq f[i] \leq n$

(C2) $\forall i, j: f[i] \neq f[j]$

A data fault may change any of the numbers such that the above constraints are not met. The goal is to detect efficiently when this happens. The violation of (C1) is easy to detect. Every node $i$ checks the value $f[i]$ periodically. If it is not between 1 and $n$, then a fault has occurred. The constraint (C2) is more interesting. At first glance it seems counter-intuitive that we can detect violation of $(\mathrm{C} 2)$ in $O(1)$ messages. However, by adding auxiliary variables, the above task can indeed be accomplished. We maintain $g[i]$ at each process $P_{i}$ such that in a legal global state the following holds:

$$
f[i]=j \equiv g[j]=i
$$

Thus, $g$ represents the inverse of the array $f$. Note that the inverse of a function exists iff it is one-one and onto which is true in this case. If each process $P_{i}$ maintains $f[i]$ and $g[i]$, then it is sufficient for a node to check periodically the following constraints:

(D1) $\forall i: 1 \leq f[i] \leq n$

(D2) $\forall i: 1 \leq g[i] \leq n$

(D3) $g[f[i]]=i$

It is easy to show that (C2) is implied by (D1)-(D3). If for some distinct $i$ and $j, f[i]$ is equal to $f[j]$, then $g[f[i]]$ and $g[f[j]]$ are also equal. This means that $(g[f[i]]=i)$ and $(g[f[j]]=j)$ cannot be true simultaneously.

(D3) can be checked by $P_{i}$ by sending a message to $P_{f[i]}$ periodically, prompting $P_{f[i]}$ to check whether $g[f[i]]=i$ is true.

Note that by introducing additional variables we have also introduced additional sources of data faults. It may happen that requirements $(\mathrm{C} 1)-(\mathrm{C} 2)$ are met, but due to faults in $g$, constraints (D1)-(D3) are not met. We believe that the advantage of local detection of a fault outweighs this disadvantage.
The above scheme has an additional attractive property: If we assume that there is a single fault in $f$ or $g$, then it can also be automatically corrected as shown next. The function $g$ being inverse of $f$ also implies that $f$ is inverse of $g$. This implies that the following constraint (D4) is also met for a fault-free data structure:

(D4) $f[g[i]]=i$

$$
\begin{array}{|l}
\begin{array}{l}
P_{i}:: \\
\text { var }
\end{array} \\
\quad f, g: \text { array[1..n] of integer; } \\
\text { Periodically do } \\
\quad \text { if }(g[f[i]] \neq i) \wedge f[g[f[i]]] \neq f[i] \\
\quad g[f[i]]=i \\
\quad \text { if }(f[g[i]] \neq i) \wedge g[f[g[i]]] \neq g[i] \\
\quad f[g[i]]=i
\end{array}
$$

Figure 5: Implementation of Permutation with local correction of 1 fault

Now assume that a node $i$ discovers that $g[f[i]] \neq i$. This means that either $f[i]$ or $g[f[i]]$ got corrupted. To detect which of the case has happened, it is sufficient to check whether

$$
f[g[f[i]]]=f[i]
$$

If the above equation does not hold, then $g[f[i]]$ is corrupted and is set back to $i$. If the above equation holds, but $g[f[i]] \neq i$ then $f[i]$ is corrupted and it needs to be reset. What value should $f[i]$ be set to? We need to set it to $k$ such that $g[k]$ equals $i$. This correction would be done by node $k$ because node $k$ will find that $f[g[k]] \neq k$. Hence, by the similar reasoning as above it will deduce that $f[g[k]]$ is corrupted and will reset it to $k$. The program for $P_{i}$ is shown in Figure 5. For simplicity, we let process $P_{i}$ simply read and write variables of other processes. In practice, this may be translated into messages. Note that in our scheme a permutation may undetectably change into another permutation (when there are multiple faults) but if $f$ is not a valid permutation, the violation will be detected.

\subsection{Constraint (R4)}

This constraint can be modeled in terms of the following constraints: 
(E1) $\forall i:(z[i] \neq 0) \Rightarrow(\operatorname{code}[z[i]]=i)$

(E2) $\forall i, j:(\operatorname{code}[j]=i) \Rightarrow(z[i] \geq j)$

For checking (E1), node $i$ prompts the node $z[i]$ to verify that code $[z[i]]=i$. If the check fails, then $z[i]$ can be set to 0 , which may not be the correct value for $z[i]$. If $z[i]$ is set incorrectly to 0 , then constraint (E2) would also be violated. As a result, while checking for (E2), $z[i]$ would be set appropriately. For checking (E2), every node $j$ sends a message to node $\operatorname{code}[j]$ to verify that $z[\operatorname{cod} e[j]] \geq j$. If (E2) is found to be violated upon receiving a message from node $j$, then $z[\operatorname{cod} e[j]]$ is set to $j$.

\subsection{Constraint (R5)}

The constraint (R5) can be checked and corrected locally.

\subsection{Complete Algorithm}

Depending upon the set of constraints $(\mathcal{R}$ or $\mathcal{C})$ that a process obeys, we have two versions of the algorithm. They differ in the guarantees about the resulting tree and their time complexities.

\subsubsection{Maintaining $\mathcal{R}$}

As we proved in Theorem 1 , the set of constraints $\mathcal{R}$ is sufficient to maintain a spanning tree. The complete algorithm for process $i$ to maintain the constraint set $\mathcal{R}$ is given in the Figure 6. We will refer to this algorithm as $S S R$. In the algorithm, instead of denoting variables like code $[i]$, we have used $P_{i}$.code to emphasize that the variables are local to the processes and are not shared. The algorithm checks the constraints one by one and on the violation of a constraint, it takes corrective action. For checking constraints which involve obtaining the value of another process's variable, we have used a primitive get. This involves the sender sending a request for the required variable and the receiver then replying with the appropriate value. So a get operation would involve two messages being exchanged. Most of the algorithm follows directly from the checks required for a constraint. The important thing to note here is that the set of constraints $\mathcal{R}$ leads us to an efficient algorithm. The formal proof of correctness of the algorithm is given in full version of this paper [GA04].

The following theorems gives the time and message complexity of this algorithm and it is the main result of this paper.
Theorem 3 The algorithm SSR requires amortized $O(1)$ time per node and amortized $O(1)$ messages per node in one asynchronous cycle.

Proof: In the algorithm SSR, each node sends a constant number of messages - one per get and one on every other send. This results in a total of $O(n)$ messages being sent in the system. The number of messages received by a process $i$ depends upon the number of times $i$ appears in the code. Assuming a random code, every node would have to process $O(1)$ messages on average. Since each node takes constant number of steps without blocking for any message, so every process requires $O(1)$ time on average to complete one asynchronous cycle.

Self-stabilizing algorithms report the stabilization time in terms of the number of asynchronous rounds required for the algorithm to stabilize. For our algorithm, due to the presence of asynchronous receives for the messages of type "Check $z$ ", this is not a good measure. For this purpose, we use the following model:

Every process starts execution at the same time. Subsequently, every computational step, message send and message receive takes one unit of time. In addition, every message takes one unit of time to travel from source to destination. The time units taken for the execution of the algorithm measure the time complexity of the algorithm.

A discussion on the relevance of this model and a comparison with the asynchronous round model is given the full version of this paper [GA04].

Theorem 4 The algorithm SSR stabilizes in $O(d)$ time in the model given above, where $d$ is the upper bound on the number of times a node appears in the code.

The proof of this theorem is also given in the full version of this paper[GA04].

The problem of choosing the first $n-2$ numbers of code at random can be considered as the problem of randomly assigning $n-2$ balls to $n$ bins. The following theorem is a standard result in probability theory [MR95]:

Theorem 5 If $n$ balls are thrown randomly in $n$ bins, then with the probability at least $1-n^{-c}$, every bin has $O((\log n) / \log \log n)$ balls. Here $c$ is any arbitrary constant.

For a randomly chosen code, this theorem provides an upper bound for $d$ and hence an upper bound on the stabilization time, with very high probability. 


\subsubsection{Maintaining $C$}

Maintaining $C$ requires ensuring $R 3(i i)$ in addition to $\mathcal{R}$. We introduce the variable $g$ and enforce the constraints (D1)-(D3) listed in section 4.2. As discussed in the Section 4.2, one error in the data structures $f$ and $g$ can be corrected using the algorithm given in Figure 5. Including this module in the algorithm SSR gives us an $O(1)$ algorithm (Figure 7) which is capable of correcting many errors in the data structures. Unfortunately, this algorithm is not able to handle more than one correlated errors in $f$ and $g$. When an error cannot be corrected by the $O(1)$ correction algorithm, the second check for the consistency of $f$ and $g$ fails for some node. This node sends out a message to every other node informing them to start the main correction algorithm.

Upon starting the main correction algorithm, every node sends out its $z$ value to node $P_{n}$. Node $P_{n}$ collects responses from every node and then establishes a mapping between the nodes which have $z=0$ and the $f$ values that have not been allocated. By allocated $f$ values, we mean the $f$ values which can be obtained as $z+1$, for some $z \neq 0$. The nodes which have $z \neq 0$ are assigned $f=z+1$. These results are communicated back to the nodes. In this case, the node $P_{n}$ would have to do $O(n)$ work. After finishing the $O(n)$ correction algorithm, the nodes switch back to the normal correction algorithm. We will refer to this complete algorithm as SSC.

Clearly, there is a trade-off involved in choosing between the two algorithms. The algorithm for maintaining $\mathcal{R}$ is more efficient but gives weaker guarantees over the resulting spanning tree than the algorithm for maintaining $C$.

\subsection{Changing the Root Node}

The algorithms SSR and SSC can be easily modified to allow the root node to change dynamically i.e. any node (not necessarily $n$ ) can become the root of the tree and the root can be changed during the operation of the algorithm. This can be achieved by changing the constraints (R2) and (R3)(i) in the following way:

$$
\begin{aligned}
& (\forall i: 1 \leq i \leq n-1 \Rightarrow 1 \leq \operatorname{code}[i] \leq n) \\
& \wedge(\operatorname{code}[n]=0)
\end{aligned}
$$

(R3)(i) $\forall i: i \neq \operatorname{code}[n-1] \Rightarrow 1 \leq f[i]<n$

The modified constraints are also easy to check and maintain. The algorithms which allow dynamic root can

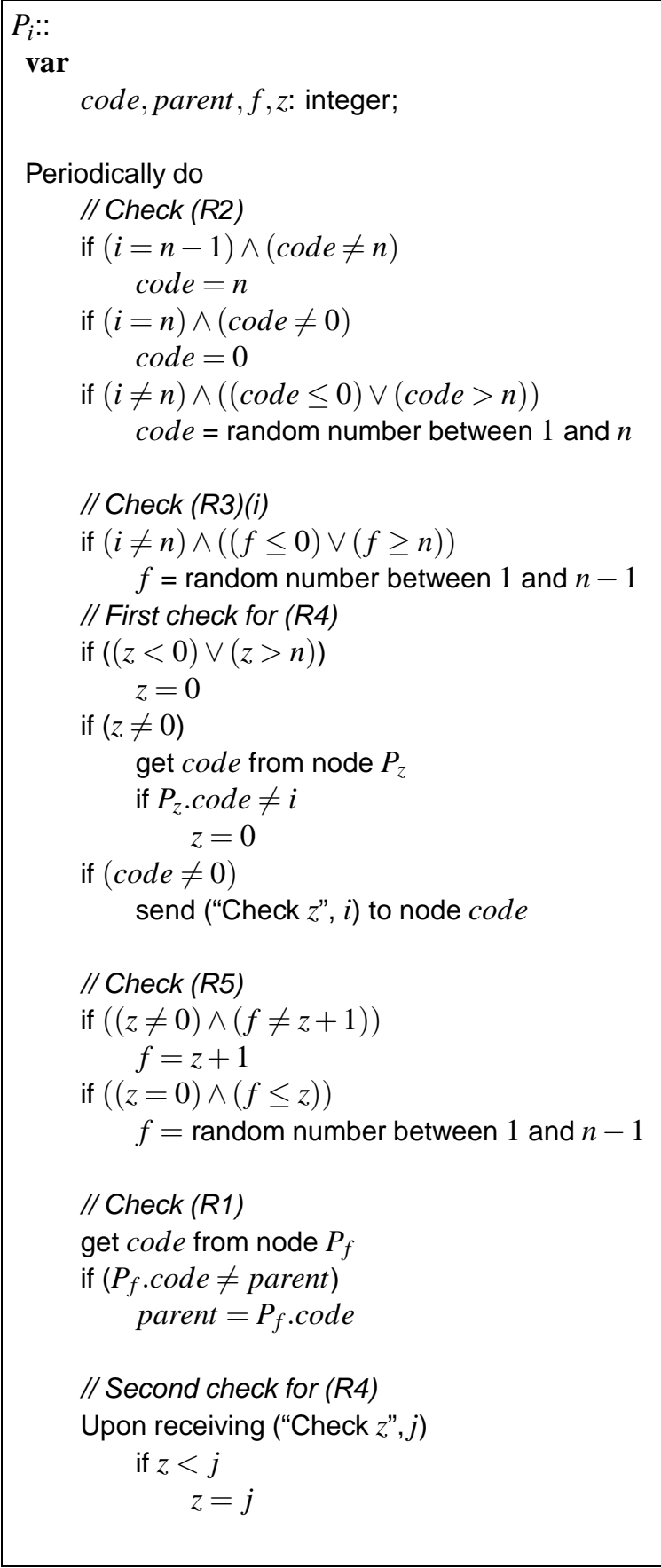

Figure 6: Algorithm SSR for maintaining the constraint set $\mathcal{R}$ 


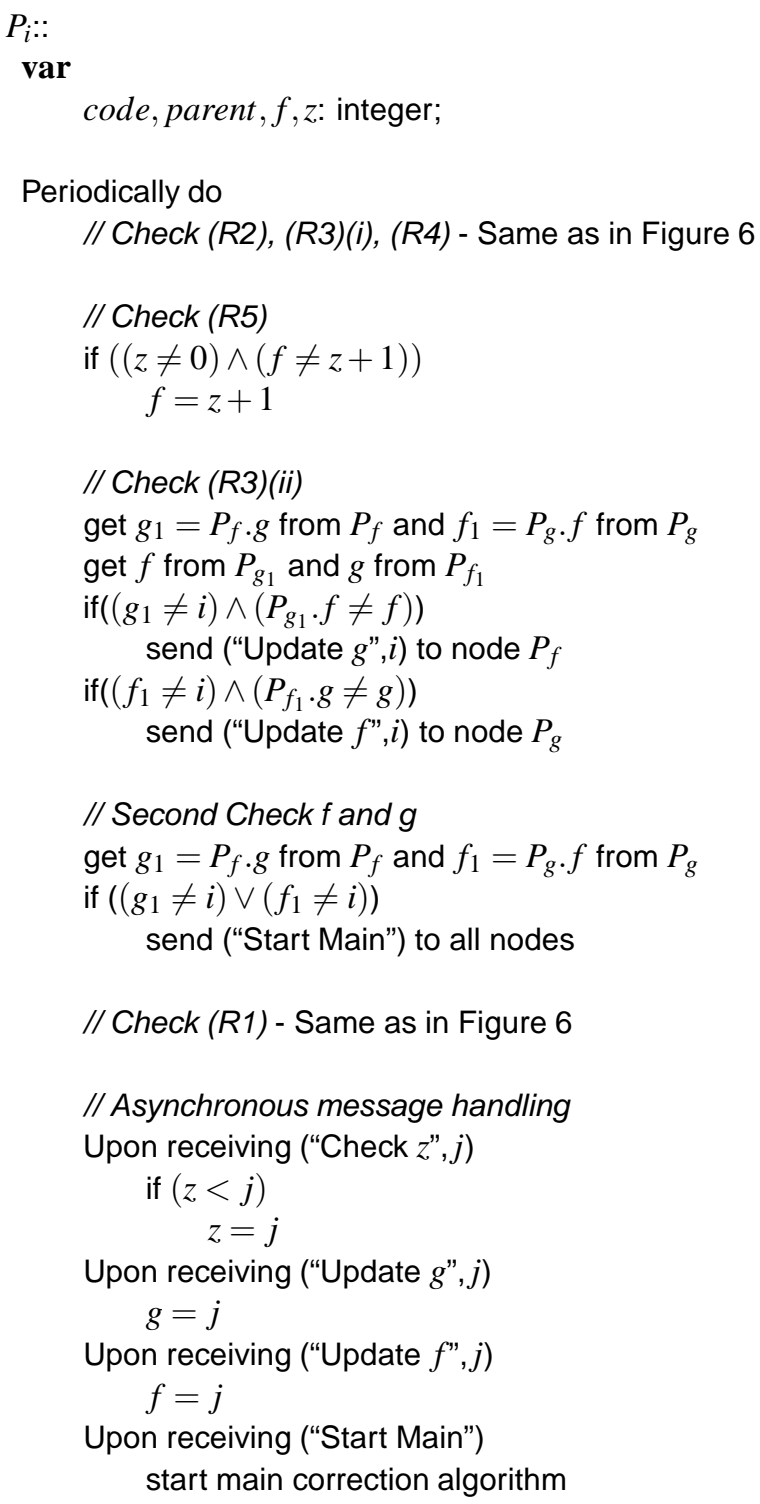

Figure 7: Algorithm SSC for maintaining the constraint set $C$ then be obtained by changing the algorithms $S S R$ and SSC to accommodate checking for these new constraints instead of the old ones. In the next section we present an application which utilizes this feature.

\section{Applications}

The algorithm for maintaining constraint set $\mathcal{R}$ ensures that if the code is changed, then the spanning tree would stabilize to reflect that change. This property of the algorithm could be used by an application to purposefully change the spanning tree from time to time. As discussed earlier, every code of length $n-1$ represents a unique tree. If we were maintaining a tree isomorphic to the code tree (by maintaining the set of constraints $C$ ), then a node wishing to change the tree could have changed its local code value. It can be proved that this would have resulted in the spanning tree being changed. But if we are just maintaining the set of constraints $\mathcal{R}$, then changing the code value at a node may not always result in a change in the tree. To get around this problem, whenever a node $i$ wishes to change the tree, it would change the value of $\operatorname{code}[f[i]]$. This changes parent $[i]=\operatorname{code}[f[i]]$ and hence the spanning tree changes. Note that this change may result in some more changes in the spanning tree as the parent of some other nodes may also get modified. Since the algorithm for maintaining the set $\mathcal{R}$ of constraints is efficient, this results in an efficient way of changing the tree. We present two applications which need to change their tree.

- Security Application: Consider a scenario in which a set of nodes are contacting each other by using a tree for routing messages. For the system's security, this tree must not be revealed to the adversary. In case a security breach is suspected or after a regular interval of time, the tree must be changed and any node should be able to initiate this change without requiring active participation from other nodes. Our algorithm provides one such way. When a node $i$ wishes to change the structure of the tree, it could just change the value of $\operatorname{code}[f[i]]$ and initiate the correction algorithm. The ability to change the root node is critical here;otherwise, the adversary could always attack the fixed root node.

- Load Balancing: Consider a scenario where a set of nodes are communicating through a spanning tree for an application like convergecast. In this case, a 
node has to do work proportional to size of its subtree which consumes resources like power, CPU etc. Since we are dealing with a completely connected topology, all the nodes are equally well connected and it is possible for a node to take up the job of another node. When a node wishes to reduce its load, it could its ask one of its child $c$ to change the value of code $[f[c]]$ and hence change its load.

\section{Discussion}

So far we had assumed the underlying graph to be completely connected. Let us now consider general graphs. Due to the nature of modern computer networks, the major overhead involved in communication using message passing is incurred at the OS level. So even if a process sends a message to another process that is more than one hop away, the message overhead can be assumed to be incurred completely at the sender and receiver. In this way any network topology with routing can be considered as a complete graph.

Our algorithm can also be modified for applications which require the parent of a node to be its 1-hop neighbor. We just add a new constraint which requires a node to check if the parent assigned to it is a 1-hop neighbor. By adding this, the detection still remains $O(1)$ but correction becomes inefficient.

\section{Conclusion and Future Work}

In this paper we presented a new technique for maintaining spanning trees using labeled tree encoding. Our method requires $O(1)$ messages per node on average in one asynchronous cycle and provides fast stabilization. It also offers a method for changing the root of the tree dynamically. We also provide examples of using the selfstabilizing algorithm for some applications not related to fault tolerance. This work also demonstrates the use of the concept of core and non-core states for designing self-stabilizing algorithms. It would be interesting to extend this work for general topology. Another research direction could be to develop similar algorithm which requires nodes to have unique labels but not neccessarily in the range 1 to $n$.

\section{References}

[AG94] A. Arora and M. Gouda. Distributed reset. IEEE Transactions on Computers, 43(9):1026-1038, 1994.

[AK93] S. Aggarwal and S. Kutten. Time optimal self-stabilizing spanning tree algorithm. In FSTTCS93 Proceedings of the 13th Conference on Foundations of Software Technology and Theoretical Computer Science, Springer-Verlag LNCS:761, pages 400-410, 1993.

[AKY91] Y. Afek, S. Kutten, and M. Yung. Memory-effi cient self stabilizing protocols for general networks. In Proceedings of the 4th International Workshop on Distributed algorithms, pages 15-28. Springer-Verlag New York, Inc., 1991.

[AS97] G. Antonoiu and P.K. Srimani. Distributed self-stabilizing algorithm for minimum spanning tree construction. In $E u$ ropean Conference on Parallel Processing, pages 480-487, 1997.

[CD94] Z. Collin and S. Dolev. Self-stabilizing depth-fi rst search Information Processing Letters, 49(6):297-301, 1994.

[Dij74] E. W. Dijkstra. Self-stabilizing systems in spite of distributed control. Communications of the ACM, 17:643-644, 1974.

[DIM89] S. Dolev, A. Israeli, and S. Moran. Self-stabilization of dynamic systems. In MCC Workshop on Self-Stabilizing Systems, 1989.

[DIM90] S. Dolev, A. Israeli, and S. Moran. Self-stabilization of dynamic systems assuming only read/write atomicity. In Proceedings of the ninth annual ACM symposium on Principles of Distributed Computing, pages 103-117. ACM Press, 1990.

[DM01] N. Deo and P. Micikevicius. Prufer-like codes for labeled trees. Congressus Numerantium, 151:65-73, 2001.

[GA04] V. K. Garg and A. Agarwal. Self-stabilizing spanning tree algorithm with a new design methodology. Technical report, University of Texas at Austin, 2004. Available as "http: //maple.ece.utexas.edu/TechReports/ 2004/TR-PDS-2004-001.ps".

[HC92] S. Huang and N. Chen. A self stabilizing algorithm for constructing breadth fi rst trees. Information Processing Letters, 41:109-117, 1992.

[Joh97] C. Johnen. Memory effi cient, self-stabilizing algorithm to construct bfs spanning trees. In Proceedings of the sixteenth annual ACM symposium on Principles of Distributed Computing, page 288. ACM Press, 1997.

[MR95] R. Motwani and P. Raghavan. Randomized Algorithms. Cambridge University Press, 1995.

[Nev53] E. H. Neville. The codifying of tree-structure. Proceedings of Cambridge Philosophical Society, 49:381-385, 1953. 